\title{
Antisynthetase Syndrome in Senegalese Patients: Report of Three Cases
}

\author{
Baïdy Sy Kane ${ }^{1 *}$, Moustapha Niasse ${ }^{2}$, Michel Assane Ndour ${ }^{1}$, Awa Cheikh Ndao1, \\ Boundia Djiba1, Mohamed Dieng1, Maïmouna Sow'1, Nafissatou Diagne1, \\ Atoumane Faye ${ }^{1}$, Souhaibou Ndongo' ${ }^{1}$, Abdoulaye Pouye ${ }^{1}$ \\ ${ }^{1}$ Department of Internal Medicine, Cheikh Anta DIOP University, Dakar, Senegal \\ ${ }^{2}$ Department of Rheumatology, Cheikh Anta DIOP University, Dakar, Senegal \\ Email: ^baidysy.kane@ucad.edu.sn
}

How to cite this paper: Kane, B.S., Niasse, M., Ndour, M.A., Ndao, A.C. Djiba, B., Dieng, M., Sow, M., Diagne, N., Faye, A., Ndongo, S. and Pouye, A. (2019) Antisynthetase Syndrome in Senegalese Patients: Report of Three Cases. Open Journal of Internal Medicine, 9, 121-128.

https://doi.org/10.4236/ojim.2019.94017

Received: October 7, 2019

Accepted: November 3, 2019

Published: November 6, 2019

Copyright $\odot 2019$ by author(s) and Scientific Research Publishing Inc. This work is licensed under the Creative Commons Attribution International License (CC BY 4.0).

http://creativecommons.org/licenses/by/4.0/

(C) (i) Open Access

\begin{abstract}
Introduction: Antisynthetase syndrome is an original entity and rare autoimmune myositis and systemic disease, characterized clinically by a wide spectrum of clinical manifestations and the presence of autoantibodies directed against aminoacyl RNAt synthetases. We describe this disease in 03 Senegalese patients. Observations: The first patient was a 49-years-old black woman who was referred in our department after 06-months of follow-up for a misdiagnosis of tuberculosis. The clinical examination revealed polyarthritis, muscle weakness, chronic cough with crackling rales at the pulmonary bases, Raynaud phenomenon and dry syndrome. The second patient, a 21-years-old black woman, had polyarthritis and a progressive muscle weakness. The clinical examination showed also cutaneous signs including an erythema on the dorsal part of the fingers and the presence of the heliotrope erythema on the eyes. The last patient was a 52 years-old black woman. His clinical examination showed polyarthritis, muscle weakness and an appearance of mechanics' hands. The creatinine phosphokinase was at $6.26 \times \mathrm{N}, 40.3 \times \mathrm{N}$ and $33.64 \times \mathrm{N}$ respectively in our patients. The chest computer tomography revealed an interstitial lung disease with a pattern of non-specific interstitial pneumonia in all three patients. The autoantibodies anti-Jo1 was also positive in all patients. The diagnosis of antisynthetase syndrome was retained with an overlap of antisynthetase and Sjögren's syndrome in the first observation. The evolution was favourable in our 03 observations with a therapeutic combination including Prednisone-Azathioprine and Kinesitherapy. Conclusion: Antisynthetase syndrome has been exceptionally reported in sub-Saharan Africa. It must be particularly mentioned in front of the triad: myositis, arthritis and interstitial lung disease. The identification of an auto-antibody directed against RNA t synthetases, particularly anti-Jo1, is essential for its diagnosis. Prognosis
\end{abstract}


is related to interstitial lung involvement. The evolution has been favourable in our patients receiving Glucorticoid-Azathioprine combination therapy.

\section{Keywords}

Antisynthetase Syndrome, Myositis, Connective Tissue Disease, Africa South of the Sahara

\section{Introduction}

The idiopathic inflammatory myopathies (IIMs), known collectively as myositis, constitute a large spectrum of clinical phenotypes. As indicated by the name, the classical clinical manifestations of IIMs, such as muscle weakness, relate to chronic inflammation in skeletal muscle. This inflammation frequently affects other organs, including the skin, joints, lungs, gastrointestinal tract and heart, indicating the systemic nature of this disease. On the basis of muscle symptoms, skin rash and histopathological features, different subgroups have been identified in IIM, including dermatomyositis, polymyositis, inclusion body myositis, immune-mediating necrotizing myopathy and overlap myositis [1] [2].

The discovery of myositis-specific autoantibodies, among which autoantibodies directed against the aminoacyl-transfer ribonucleic acid (tRNA) synthetases also known as antisynthetase antibodies (ASAs), has made it possible to individualize the antisynthetase syndrome (ASS) within the sub-group of overlap myositis [2] [3] [4].

To date, eight different ASAs have been described, five of which are routinely tested for by immunoblotting [5]. Anti-Jol antibody is the first to have been described and is the most commonly found [5]. ASAs constitute one of the key laboratory features of ASS, which is defined as an association of inflammatory myopathy, diffuse interstitial lung disease, joint involvement, fever and cutaneous signs such as mechanic's hands and Raynaud's syndrome [3] [5] [6].

IIM, and ASS are rarely reported among black Africans [7] [8] [9] [10] [11].

We discuss 03 Senegalese cases of ASS and we hope that these observations will add to medical literature and aid in early diagnosis of other patients presenting with similar features.

\section{Observations}

The first patient, a 49 year-old black woman, was referred to our department for management of a chronic cough associated with arthromyalgia. For the past year, his complaints were an inflammatory arthralgia of the shoulders, elbows, wrists, knees and small joints of the hands associated with diffuse myalgia and chronic dry cough. This symptomatology occurred in a context of prolonged fever and altered general condition with asthenia and anorexia.

Before her admission to our department, she had been followed for a misdiagnosis of pulmonary tuberculosis, without any bacteriological evidence, for 6 
months.

It was sent to our hospital, one month after the end of the tuberculosis treatment, if there was no improvement.

Upon his admission to our institution, we noted a persistence of arthralgia and cough. There were dry eyes and mouth, Raynaud's phenomenon and muscle weakness.

The clinical examination revealed a fever of $38.5^{\circ} \mathrm{C}$, crackling rales at the pulmonary bases, synovitis of the wrists and the knees. The muscle examination noted a symmetrical bilateral proximal muscle weakness predominating at the deltoid, psoas and quadriceps groups. Schirmer's test was positive.

The blood count showed white blood cells at $19.4 \mathrm{Giga} / \mathrm{l}$, hemoglobin at 12.8 $\mathrm{g} / \mathrm{dl}$ and platelets at $428 \mathrm{G} / \mathrm{l}$. The C-reactive protein was $480.5 \mathrm{mg} / \mathrm{l}$, Aspartate transferases (AST) at $54.4 \mathrm{UI} / \mathrm{L}(\mathrm{N}<40)$, Alanine transferases (ALT) at $26 \mathrm{UI} / \mathrm{L}$ $(\mathrm{N}<35)$ and creatinine phosphokinase $(\mathrm{CK})$ at $1032 \mathrm{UI} / \mathrm{L}(6.26 \mathrm{~N})$. Cytobacteriological examination of sputum isolated Pseudomonas aeruginosa.

The remainder of biological assessment was normal including serum creatinine, serum urea, proteinuria, acid-fast bacillus search in sputum, cytobacteriological examination of urine, blood cultures, HBs antigen and HIV serology were negative.

The chest computer tomography (CT) showed an interstitial lung disease (ILD) with a pattern of non-specific interstitial pneumonia (NSIP) (Figure 1).

Immunologically the autoantibodies against-Jo1 $(>8 \mathrm{UI} / \mathrm{ml})$ and autoantibodies against-SSA antibodies $(7.2 \mathrm{UI} / \mathrm{ml})$ were positive. The other autoantibodies against SSB, U1-RNP, cyclic citrullinated peptide (CCP) and rheumatoid factors (RF) were negative.

The diagnosis of ASS associated Sjögren's syndrome and complicated by bronchopulmonoray infectious was retained.

The evolution was favourable under antibiotic therapy in the first time followed by a combination therapy including prednisone at $1 \mathrm{mg} / \mathrm{kg} / \mathrm{day}$ (and adjuvants), azathioprine (AZT) at $2 \mathrm{mg} / \mathrm{kg} /$ day and kinesitherapy.

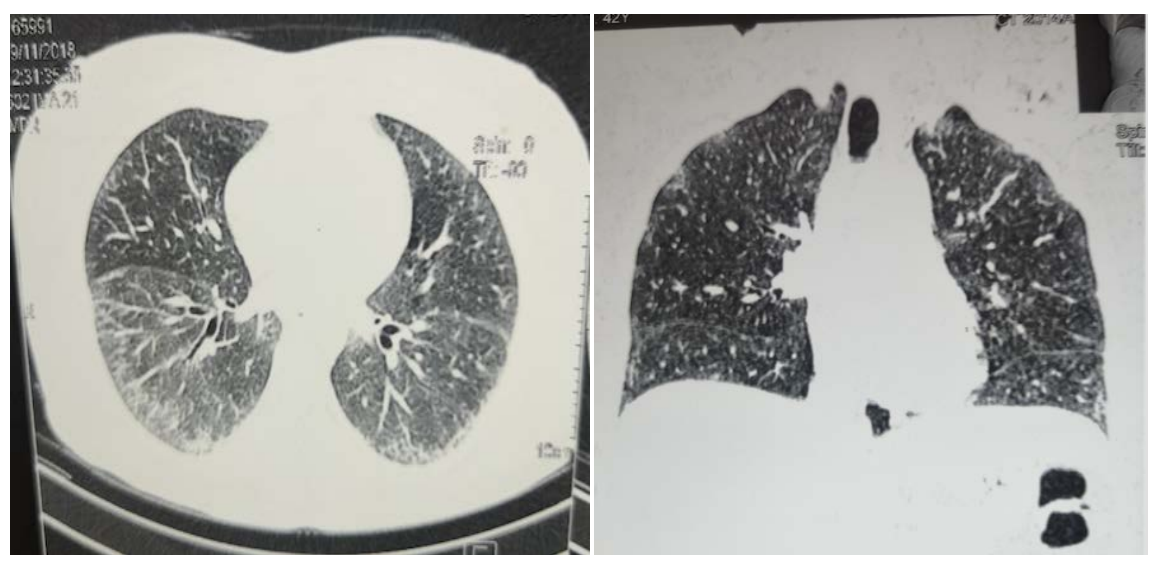

Figure 1. Chest computer tomography: Interstitial lung disease in our patient. Presence of bilateral ground glass areas mainly in the sub-pleural region with some traction bronchiectasis and diffuse interstitial micronodules. 
The second patient was 21 years-old black woman and was referred to our department for the management of polyarthritis associated with progressive proximal muscle weakness. There was no significant clinical history. The clinical assessment revealed arthralgias that had progressed over the past 02 months involving the shoulders, elbows, wrists and knees. A month later, muscle weakness appeared with difficulty in getting up from the sitting position and walk also and exertional dyspnea and asthenia.

There was a fever; the general condition was relatively preserved.

Upon admission, the physical examination noted synovitis of the wrist and knee elbows and proximal muscle deficiency. There was an erythema on the hands and a heliotrope erythema. There was no Raynaud's phenomenon, the pulmonary auscultation as well as the rest of the normal examination.

The blood count showed the white blood cells at $19.6 \mathrm{G} / \mathrm{l}$, hemoglobin at 10.2 $\mathrm{g} / \mathrm{dl}$ with platelets at $369 \mathrm{G} / \mathrm{l}$. The C-reactive protein was $23.92 \mathrm{mg} / \mathrm{l}$, ALT at 626 $\mathrm{UI} / \mathrm{L}(\mathrm{N}<35)$, AST at $1326 \mathrm{UI} / \mathrm{L}(\mathrm{N}<40)$ and CK $6660 \mathrm{UI} / \mathrm{L}(40.3 \mathrm{~N})$. The remainders of biological assessment including creatinine serum, blood urea, albuminemia, proteinuria, HBsAg, HIV serology were negative.

The chest CT concluded at NSIP.

Immunologically, the anti-Jol antibodies $(>8 \mathrm{UI} / \mathrm{ml})$, anti-SSB antibodies ( $>8$ $\mathrm{UI} / \mathrm{ml}$ ) and rheumatoid factor were positive. The other autoantibodies anti-SSA, anti-U1-RNP and anti-CCP were negative.

The diagnosis of an ASS has been retained.

The evolution was favourable under a combination of prednisone $1 \mathrm{mg} / \mathrm{kg} /$ day (and adjuvants), AZT at $2 \mathrm{mg} / \mathrm{kg} /$ day and physiotherapy.

The last patient was 52 years-old woman, she referred to our hospital a diagnostic of polyarthritis associated with muscle weakness. High blood pressure was his only medical history.

She reported inflammatory arthralgia of the wrists and knees and progressive muscle weakness.

The clinical examination revealed a more pronounced proximal muscle deficit in the lower limbs with quadricipital amyotrophy and hyperkeratosis of the hands or "mechanics' hands" (Figure 2). There was no synovitis, no Raynaud's phenomenon and pulmonary auscultation was normal.

The blood count showed white blood cells at $11.9 \mathrm{G} / \mathrm{l}$, hemoglobin was 13.9 $\mathrm{g} / \mathrm{dl}$ and platelets at $420 \mathrm{G} / 1$.

The C-reactive protein was $91.9 \mathrm{mg} / \mathrm{l}, \mathrm{AST}$ at $230 \mathrm{IU} / \mathrm{L}(6.8 \mathrm{~N}), \mathrm{ALT}$ at 216 $\mathrm{IU} / \mathrm{L}(4 \mathrm{~N})$ and $\mathrm{CK}$ at $5652 \mathrm{IU} / \mathrm{L}(33.64 \mathrm{~N})$.

The remainder of biological investigations including creatinine serum, blood urea, protidema, albuminemia, proteinuria, HBsAg, and HIV serology were negative.

The chest CT shows an ILD with a pattern of NSIP.

Immunologically, anti-Jol antibodies $(>8 \mathrm{UI} / \mathrm{ml})$, anti-SSA antibodies $(>8$ $\mathrm{UI} / \mathrm{ml}$ ) were positive. The other autoantibodies antinuclear antibodies, anti-SSB, anti-centromeres, anti-Scl 70, RF, anti-U1-RNP and anti-CCP were negative. 


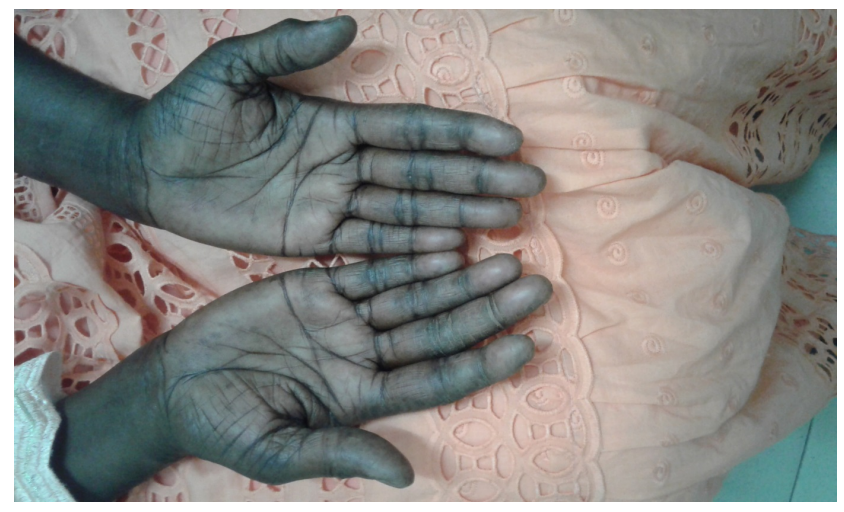

Figure 2. Mechanic's hand in our patient. Hyperkeratosis of the fingers associated with fissures and hyperpigmentation.

The diagnosis of an ASS has been retained.

The evolution was favourable under a combination of prednisone $1 \mathrm{mg} / \mathrm{kg} /$ day (and adjuvants), AZT at $2 \mathrm{mg} / \mathrm{kg} /$ day and physiotherapy.

\section{Discussion}

The first case of ASS was described in by Margurie et al., in 1990 [12].

This syndrome is an original entity and rare autoimmune systemic disease, characterized clinically by a wide spectrum of clinical manifestations and the presence of autoantibodies directed against aminoacyl RNAt synthetases [2] [13] [14].

The annual incidence of ASS can be considered to be around 0.6 per 100,000 individuals. It affects women and men in an approximate ratio of 2:1, and the mean age at diagnosis is 50 years [12]. It has been rarely reported in sub-Saharan Africa [8]. On a large multicenter study about systemic diseases in Senegal, we described only three cases of ASS in 10 years [11].

We have described 03 observations of ASS in Senegalese women.

The pathogenesis of this myositis remains unclear [12]. The initiation of the disease seems to arise in a multifactorial context, with first lesions occurring within the lungs. This may lead to aberrant self-antigen exposure, tolerance breakdown and abnormal activation of both innate and adaptive immunity, resulting in the patients with favourable genetic background to autoimmune-mediated organ lesions [15]. Although several associations between MHC class II alleles and certain MSAs have been reported, the best established association being that between anti-Jo1 autoantibodies, the most prevalent autoantibodies against RNAt synthetases, and the HLA-DRB1*03:01 [16].

Since his first description this disease was characterized clinically by the association of myositis, interstitial lung disease, cutaneous hyperkeratosis/Mechanics' hands, joint involvement, fever and Raynaud's phenomenon [2] [13] [14]. In most cases of the literature, ASS includes at least inflammatory myositis and/or interstitial lung disease (ILD). Other non-specific features are quite commonly reported, including polyarthritis, mechanic's hands, and Raynaud's phenome- 
non. Involvement of other organs is less frequently described, but includes heart (pericarditis, myocarditis) or digestive (gastro-oesophageal reflux, dysphagia) involvement [17]. The presentation is rarely complete, in our observations, the triad: myositis, interstitial lung disease and polyarthritis was constant and should lead to the diagnosis of ASS. The fever was noted in our 02 observations; the Raynaud phenomenon and "mechanic's hands" concerned only one patient. Diagnostic difficulties may be related to its inaugural mode, in the absence of muscle weakness, it may be confused with other inflammatory rheumatic diseases or other causes of interstitial lung disease (ILD). It is necessary to pay attention to the diagnosis of ASS even in cases with isolated manifestations of ILD, arthritis, or myositis [12]. In our first observation, the pulmonary involvement was inaugural and in our context of tuberculosis (TB) endemicity, the patient had been managed for a misdiagnosis of TB. Berthe et al. also reported in their case report of ASS, an initial diagnosis confusion with TB [8]. A clinical identification of mechanic's hands is also essential particularly in cases amyopathic disorders. The main differential diagnosis is eczema (which may be hyperkeratotic and fissured) and palmar psoriasis with fissures. However, eczema is also pruritic and psoriasis is rarely limited in the fingers. Evocative lesions of psoriasis could be found in the rest of the skin as well as a familial history of psoriasis [5].

To date, eight different ASAs have been described. The most common anti-ARS antibody recognizes Jol (histidyl-tRNA synthetase) and was first identified in 1980. Anti-Jo1 autoantibodies can be identified in up to $19 \%$ of adult patients with IIM and the remaining anti-ARS antibodies, namely anti-PL7 (anti-threonyl-tRNA synthetase), anti-PL12 (anti-alanyl-tRNA synthetase), anti-EJ (anti-glycyl tRNA synthetase), anti-OJ (anti-isoleucyl-tRNA synthetase), anti-Ha (anti-tyrosyl-tRNA synthetase), anti-KS (anti-asparagyl-tRNA synthetase) and anti-Zo (anti-phenylalanyl-tRNA synthetase) antibodies, are collectively found in a further $3.5 \%$ [16].

Anti-Jo1 was positive in all of our three patients. Apart from our third observation, these were the only ASAs that were investigated in our patients, due to the limitations of the means and these constituted a limitation of our work. However, ASAs are mutually exclusive [15]. Furthermore, the disease presentation varies depending on which ASAs are present. Muscle disease is common in patients with anti-Jo1, anti-PL7 or anti-EJ antibodies, and arthritis even more so in those with anti-Jo1 antibodies, whereas the presence of anti-PL7, anti-KS, anti-OJ or anti-PL12 antibodies is associated with more prevalent or more severe ILD [16].

The prognosis of ASS depends on the presence or absence of pulmonary involvement, which may be complicated by pulmonary fibrosis, pulmonary arterial hypertension and acute respiratory distress [5].

ILD is a major contributor to morbidity and mortality [18].

The strongest predictor of ILD in patients with ASS is the presence of anti-Jo-1 antibodies. Nearly $70 \%$ of patients with ASS with ILD have detectable an- 
ti-Jo- 1 antibodies [3].

ILD must be systematically detected during myositis and was constant in our 03 observations. We suggest to screen for ASAs in patients with NSIP, the most common HCRT pattern of ILD and that we have identified in our patients.

Treatment of ASSs is challenging and based upon small case series rather than randomised, clinical trials. It is generally agreed that corticosteroids are the first line therapy with the addition of other immunosuppressants, such as mycophenolate, methotrexate (MTX), AZA or cyclophosphamide, but there is no consensus on which immunosuppressive agents to prefer. The choice depends primarily on the severity of the disease [18]. Rituximab has been also successfully used in the literature. The combination of Glucocorticoid (GC) and MTX (GC-MTX) or AZT (GC-AZT) is often used in first line therapy. GC-AZT combination was administrated to our 03 patients. AZT therapy might be preferable to MTX or patients with liver disease, patients unwilling to abstain from alcohol or patients with severe ILD [19].

\section{Conclusion}

SAS has been exceptionally reported in sub-Saharan Africa. It must be particularly mentioned in front of the triad: myositis, arthritis and interstitial lung disease. The identification of an auto-antibody directed against RNA t synthetases, particularly anti-Jo1, is essential for its diagnosis. Prognosis is related to interstitial lung involvement. The evolution has been favourable in our patients receiving GC-AZT combination therapy. A multicenter study should be conducted to improve our knowledge of this disease in black African patients.

\section{Declarations}

\section{Consent for Publication}

Written informed consent for publication was obtained from the patients.

\section{Funding}

The authors declare that they have not received another source of funding.

\section{Conflicts of Interest}

The authors declare that they have no competing interests.

\section{References}

[1] Lundberg, I.E., Visser, M.D. and Werth, V.P. (2018) Classification of Myositis. Nature Reviews Rheumatology, 14, 269-278. https://doi.org/10.1038/nrrheum.2018.41

[2] Meyer, A. and Sibilia, J. (2018) Strategy for Suspected Myositis. Revue du Rhumatisme, 85S, A23-A29. https://doi.org/10.1016/S1169-8330(18)30172-8

[3] Badshah, A., Haider, I., Pervez, S. and Humayun, M. (2019) Antisynthetase Syndrome Presenting as Interstitial Lung Disease: A Case Report. Journal of Medical Case Reports, 13, 241. https://doi.org/10.1186/s13256-019-2146-0 
[4] Meyer, A., Lannes, B., Goetz, J., Echaniz-Laguna, A., Lipsker, D., Arnaud, L., et al. (2018) Inflammatory Myopathies: A New Landscape. Joint Bone Spine, 85, 23-33. https://doi.org/10.1016/j.jbspin.2017.03.005

[5] Gusdorf, L., Morruzzi, C., Goetz, J., Lipsker, D., Sibilia, J. and Cribier, B. (2019) Mechanics Hands in Patients with Antisynthetase Syndrome: 25 Cases. Annales de Dermatologie et de Vénéréologie, 146, 19-25. https://doi.org/10.1016/j.annder.2018.11.010

[6] Bouardi, N.E., Alaoui, A., Haloua, M., Lamrani, Y., Boubbou, M., Maaroufi, M. and Alami, B. (2019) Diffuse Interstitial Lung Disease Revealing Antisynthetase Syndrome: About Two Cases. The Pan African Medical Journal, 32, 95. https://doi.org/10.11604/pamj.2019.32.95.17819

[7] Adelowo, O.O. and Bello, M.K.N. (2014) Systemic Autoimmune Diseases: Not So Rare in Black Patients. Rheumatology (Sunnyvale), 4, 130.

https://doi.org/10.4172/2161-1149.1000130

[8] Berthe, A., Diop, M.M., Touré, P.S., Gueye, D., Faye, F.A., Diop, B.M. and Ka, M.M. (2017) Antisynthetase Syndrome Simulating a Pulmonary Tuberculosis. Revue Africaine de Médecine Interne, 4, 53-56.

[9] Diallo, M., Fall, A.K., Diallo, I., Diédhiou, I., et al. (2010) Dermatomyositis and Polymyositis: 21 Cases in Senegal. Médecine Tropicale, 70, 166-168.

[10] Dieng, M.T., Diallo, M., Dia, D., Sow, A. and Ndiaye, B. (2005) Dermatomyositis in Senegal: Study of 56 Cases. Dakar Médical, 50, 123-127.

[11] Kane, B.S., Niasse, M., Ndiaye, A.A., Ndao, A.C., et al. (2018) Systemic Diseases in Dakar (Senegal): Spectrum, Epidemiological Aspect and Diagnostic Time-Limit. Open Journal of Internal Medicine, 8, 196-206. https://doi.org/10.4236/ojim.2018.83019

[12] Marin, F.L. and Sampalo, H.P. (2019) Antisynthetase Syndrome and Autoantibodies: A Literature Review and Report of 4 Cases. American Journal of Case Reports, 20, 1094-1103. https://doi.org/10.12659/AJCR.916178

[13] Noguchi, E., Uruha, A. and Nishino, I. (2017) Skeletal Muscle Involvement in Antisynthetase Syndrome. JAMA Neurology, 74, 992-999. https://doi.org/10.1001/jamaneurol.2017.0934

[14] Meyer, A., Lefevre, G., Bierry, G., Duval, A., Ottoviani, S., Meyer, O., et al. (2015) In Antisynthetase Syndrome, ACPA Are Associated with Severe and Erosive Arthritis. Medicine, 94, e523. https://doi.org/10.1097/MD.0000000000000523

[15] Gallay, L., Gayed, C. and Hervier, B. (2018) Antisynthetase Syndrome Pathogenesis: Knowledge and Uncertainties. Current Opinion in Rheumatology, 30, 664-673. https://doi.org/10.1097/BOR.0000000000000555

[16] McHugh, N.J. and Transley, S.L. (2018) Autoantibodies in Myositis. Nature Reviews Rheumatology, 14, 290-302. https://doi.org/10.1038/nrrheum.2018.56

[17] Hervier, B. and Benveniste, O. (2013) Clinical Heterogeneity and Outcomes of Antisynthetase Syndrome. Current Rheumatology Reports, 15, 349. https://doi.org/10.1007/s11926-013-0349-8

[18] Jensen, M.L., Lokke, A., Hildberg, O., Hyldgaard, C., Bendstrup, E. and Tran, D. (2019) Clinical Characteristics and Outcome in Patients with Antisynthetase Syndrome Associated Interstitial Lung Disease: A Restrospective Cohort Study. European Clinical Respiratory Journal, 6, Article ID: 1583516. https://doi.org/10.1080/20018525.2019.1583516

[19] Oddis, C.V. and Aggarwal, R. (2018) Treatment in Myositis. Nature Reviews Rheumatology, 14, 619. https://doi.org/10.1038/s41584-018-0086-5 